\title{
panorama europeo actual del control de calidad del hormigón
}

ALVARO GARCIA MESEGUER, Profesor de Investigación

\begin{abstract}
sinopsis Se presenta con cierto detalle el estado actual del tema, a partir de los trabajos en curso del Comité Editorial CEB-CIB-FIP-RILEM sobre Control Estadístico de Calidad del Hormigón. De forma comparativa, se presenta también el modus operandi recientemente introducido en España por la nueva Instrucción del Hormigón EH 73.

El artículo termina comentando la influencia que tiene un descenso de resistencia del hormigón en la seguridad de la estructura, tema éste más complejo de lo que puede parecer

$073-5$ a primera vista.
\end{abstract}

\section{INTRODUCCION}

Tanto las resistencias de los materiales como las tensiones a que éstos se ven sometidos en una estructura, son variables aleatorias. Por ello, el proyecto de estructuras se basa hoy en métodos probabilistas, abandonándose cada vez más los métodos deterministas.

En principio, si el proyectista conoce la distribución de probabilidades de las resistencias y de las solicitaciones, puede calcular la probabilidad de fallo de una estructura.

Si se consideran, por una parte, las consecuencias técnicas, económicas y sociales de un fallo estructural, y por otra, el coste asociado a la disminución de la probabilidad de fallo, se puede llegar al establecimiento de un nivel óptimo para la probabilidad de fallo de una determinada estructura. Por consiguiente, habrá que especificar los materiales en términos de parámetros de probabilidad, con objeto de asegurar que no se excede aquella probabilidad de fallo.

Estos principios, someramente enunciados, son válidos cualquiera que sea el fallo que se contemple (hundimiento, fisuración excesiva, deformación excesiva, etc.). Su aplicación a la práctica del proyecto no puede hacerse hoy en forma rigurosa, por falta de conocimientos. Pero desde un punto de vista práctico, las Normas modernas introducen esta filosofía a través del concepto de resistencia característica de los materiales y de los coeficientes parciales de seguridad relativos a acciones y materiales.
Ahora bien, una vez proyectada una estructura, ésta debe construirse, lo que entraña una serie de procesos (fabricación, transporte, colocación, compactación y curado del hormigón; encofrado; fabricación y colocación de acero, etc.) que están sujetos a variaciones, de forma tal que su resultado no puede predecirse exactamente. Además, la información que podemos obtener de tales procesos proviene, a su vez, de otros procesos de muestreo y ensayo que también están sometidos a variaciones aleatorias.

Ante estas incertidumbres que acompañan a la información, resulta necesario establecer unos criterios de decisión con objeto de interpretar debidamente los resultados disponibles. Tales criterios deben tender a que la evaluación del riesgo inicialmente prevista en fase de proyecto, no resulte superada en la fase final de construcción.

Importa, por consiguiente, establecer un cuidadoso control de calidad y, en particular, un control de calidad del hormigón, que es lo que aquí nos ocupa. E importa subrayar, desde ahora, que dicho control puede influir sobre la probabilidad de fallo mucho más acusadamente que los refinamientos de cálculo u otros aspectos de la fase de proyecto.

\section{SITUACION DEL TEMA EN EL. EXTRANJERO}

Hasta épocas recientes el control de calidad se confiaba a la pericia y vigilancia del técnico director de obra, actuando con arreglo 
a su buen sentido y criterio personal. Hoy día, en que las técnicas estadísticas de control de calidad han alcanzado gran desarrollo en otros sectores industriales, se tiende a que la construcción asimile dichas técnicas y las adapte a sus problemas propios.

En los últimos quince años la construcción en hormigón ha experimentado una verdadera revolución en sus aspectos teóricos de diseño y de cálculo, es decir, en fase de proyecto. Se han dedicado grandes esfuerzos a mejorar el conocimiento del comportamiento resistente de las piezas de hormigón y puede decirse que hoy día se dispone de procedimientos de cálculo plenamente satisfactorios. En particular, la elaboración y puesta a punto de la teoría de los Estados Límites y la desaparición de la tradicional separación entre hormigón armado y hormigón pretensado (hoy día se habla de "hormigón estructural", material que va del uno al otro sin solución de continuidad, extendiéndose incluso al hormigón en masa) son dos ejemplos del avance a que nos referimos.

Pero ese avance, repetimos, se refiere al proyecto. Y sólo muy recientemente, desde hace menos de cinco años, se está dedicando atención al control de calidad, como último eslabón imprescindible dentro de la concepción estadística que hoy se tiene del fenómeno constructivo.

En esta línea existe un Comité Mixto CEBCIB-FIP-RILEM que está estudiando el tema del control de calidad del hormigón a escala internacional. En este Comité, que fue creado en 1968, participan expertos europeos y americanos, tres por cada una de las Asociaciones representadas. Inicialmente, el Comité creó tres Grupos de Trabajo, sobre los temas: "Variabilidad del Hormigón", "Estadísticas y Reglamentos" y "Estructuras", siendo el más activo el primero de ellos.

Posteriormente, en 1971, se creó un Comité Editorial para que, aprovechando lo efectuado hasta entonces, tratase de establecer un documento de síntesis conteniendo unos criterios que pudiesen servir de guía. La tarea no era fácil, dada la disparidad de opiniones, pero se está cerca del final, ya que en marzo de 1974 este Comité habrá terminado su labor y presentará su propuesta al Comité Mixto, que se reunirá en el mes de mayo.

A título de ejemplo de disparidad de opiniones, diremos que a la pregunta: ¿qué probabilidad de aceptación debe tener un hormigón que presenta un $5 \%$ de defectuosos? (es de- cir, cuya resistencia característica real coincide con la especificada), las respuestas van desde un $5 \%$ (Dinamarca) hasta un $95 \%$ (Inglaterra), pasando por un $50 \%$ (España) $y$ un $75 \%$ (Alemania).

La mayor dificultad estriba en el hecho de que, como todos los aspectos están interrelacionados, no es posible analizar el problema separadamente, sino en su conjunto. Trataremos de explicar más esta idea.

\section{CRITERIOS DE ACEPTACION}

La probabilidad de fallo del hormigón realmente colocado en una estructura es una combinación de las tres probabilidades siguientes:

a) la probabilidad de que un hormigón idéntico al especificado, falle bajo las solicitaciones reales;

b) la probabilidad de que se suministre un hormigón idéntico al especificado; y

c) la probabilidad de que sea aceptado un hormigón idéntico al especificado.

La probabilidad a) viene influida por el criterio del proyectista; la b), por el criterio del suministrador, y la c), por el criterio de aceptación que establezcan las Normas.

No existen hoy día valores concretos para la probabilidad a), pero indirectamente viene fijada por los valores de los $\gamma$ empleados en el proyecto. En cuanto a la probabilidad b), depende del coste de producción de un buen hormigón, comparado con los premios o castigos asociados a la aceptación o rechazo. Del análisis comparado de ambos costes, cada suministrador deducirá una estrategia de producción de hormigón.

Como se ve, la interrelación es evidente. En particular, es clara la que existe entre los valores de los $\gamma$ y el criterio de aceptación-rechazo que se adopte.

El tema se dificulta más todavía al advertir que "rechazo" no significa demolición, sino simplemente "no aceptación automática». Si se produce un rechazo se abre un proceso de acciones, más o menos complicadas en función de los nuevos resultados que se vayan obteniendo, que pueden desembocar en la aceptación limpia, la aceptación con penalización, el refuerzo o la demolición.

El objetivo de conseguir una estructura correcta puede alcanzarse mediante combina- 
ciones muy diferentes de reglas relativas a proyecto, fabricación y aceptación. Así, por ejemplo, pueden combinarse reglas muy estrictas de fabricación con criterios relativamente amplios de aceptación y consecuencias menores en caso de rechazo. O viceversa, pocas reglas de fabricación (que dejarán gran libertad al suministrador) con criterios severos de aceptación y consecuencias mayores en caso de rechazo.

Todo lo dicho explica el que sea difícil establecer reglas generales válidas para todos los países. Y que sea difícil, incluso, comparar las normas de uno y otro país, puesto que responden en general a filosofías diferentes. En tales filosofías influyen, además, las costumbres imperante en cuanto a responsabilidad, que tampoco coinciden.

El proyecto, la producción y la aceptación son funciones separadas y distintas. En muchos casos la responsabilidad de cada una de esas funciones corresponde a diferentes autoridades. En otros casos las tres funciones competen a una sola autoridad. Por ello, al comparar distintos Códigos que hablan de la responsabilidad del "ingeniero" debe prestarse atención a cuál de las funciones indicadas se están refiriendo.

En lo que sigue expondremos aquellas ideas que parecen gozar de una aceptación mayoritaria por el momento. Pero debe quedar bien entendido que se trata de un tema aún en sus comienzos, que habrá de sufrir sin duda modificaciones en el futuro hasta conseguir un consenso general.

\section{4}

\section{EL CONTROL Y LA SEGURIDAD}

Toda estructura de hormigón armado, una vez construida, presenta multitud de características que difieren de las proyectadas: las armaduras no están exactamente en la posición definida en los planos; el hormigón no tiene exactamente la resistencia especificada; las dimensiones de las piezas no coinciden exactamente con las previstas, etc.

El grado de concordancia de la estructura real con la proyectada es un índice de la calidad de la ejecución de aquélla. Cuanto más alto sea el control, mayor será dicho índice, más fielmente se cumplirán las hipótesis supuestas por el proyectista y, por consiguiente, los coeficientes de seguridad reales que presente la estructura se aproximarán más a los teóricos. Por el contrario, en una obra poco controlada las desviaciones serán grandes y, en consecuencia, se verán mermados los márgenes reales de seguridad.
Existe, por tanto, una relación entre la seguridad real de una estructura y el control ejercido durante la construcción de la misma. Si el proyectista impone para la ejecución un control (1) cuidadoso y sistemático, podrá utilizar en sus cálculos valores más afinados para los coeficientes parciales de seguridad $\gamma$; contrariamente, el proyectista podrá tolerar controles de ejecución menos cuidados si, habiéndolo previsto en sus cálculos, se ha cubierto mediante el oportuno aumento de los coeficientes $\gamma$. En ambos casos, el margen de seguridad real de la estructura construida será aproximadamente el mismo.

Esta relación entre los valores de $\gamma$ y el grado de control está puesta de manifiesto en las Recomendaciones Internacionales CEB-FIP de 1970 , aunque sin cuantificar. Pero hasta el momento, ningún Reglamento nacional la ha incorporado, quizá porque la idea es considerada como revolucionaria. En España, no obstante, se ha dado el paso, y la Instrucción EH 73 establece unos valores numéricos para $\gamma_{c}, \gamma_{s}$ y $\gamma_{f}$ que son función de las condiciones de control del hormigón $\left(\gamma_{c}\right)$, del acero $\left(\gamma_{s}\right)$ y de la ejecución $\left(\gamma_{f}\right)$ (2).

Como se ve en la figura 1 , el coeficiente $\gamma_{c}$ puede variar entre 1,40 y 1,70 según el nivel de control. Antes de definir estos niveles y sus limitaciones, importa subrayar que esta forma de enfocar el tema significa que el proyectista debe especificar a priori el nivel de control (1) que haya de utilizarse en obra,

\begin{tabular}{c|c|c}
\hline $\begin{array}{c}\text { Nivel } \\
\text { de control }\end{array}$ & $\mathbf{f}_{c k}$ & $\gamma_{\mathrm{c}}$ \\
\hline INFERIOR & $\leqslant 150 \mathrm{kp} / \mathrm{cm}^{2}$ & 1,70 \\
\hline INTERMEDIO & $\leqslant 250 \mathrm{kp} / \mathrm{cm}^{2}$ & 1,50 \\
\cline { 2 - 2 } & IN SITU & 1,50 \\
\hline SUPERIOR & PREFABRICACION & 1,40 \\
\hline
\end{tabular}

Fig. 1

(1) Nos referimos a control de aceptación, no a control de producción. La confusión entre ambos origina malos entendidos, sobre todo entre per. sonas de distinto idioma nativo.

(2) La notación empleada en este artículo es la del Sistema Internacional Unificado CEB - FIP - ACI, adoptada oficialmente en España. 
pues este dato condiciona el valor de $\gamma_{c}$ que él deba utilizar en sus cálculos

Las definiciones de los niveles de control en España, son las siguientes:

\section{Nivel inferior o reducido}

En este nivel, la resistencia del hormigón no se controla directamente, es decir, no se confeccionan probetas. El único control que se efectúa es el del hormigón fresco.

Este nivel inferior puede emplearse en obras de pequeña importancia, teniendo en cuenta las siguientes observaciones:

- la resistencia característica de proyecto $\left(\mathbf{f}_{c k}\right)$ no debe superar los $150 \mathrm{kp} / \mathrm{cm}^{2}$;

- el coeficiente de minoración del hormigón $\left(\gamma_{c}\right)$ debe considerarse igual a 1,70;

- se emplearán dosificaciones tipo previamente establecidas, con un contenido en cemento no inferior a $300 \mathrm{~kg} / \mathrm{m}^{3}$;

- se extremará la vigilancia de las operaciones de dosificación, amasado, puesta en obra, etc., y se llevará un control sistemático del asiento en cono de Abrams, con no menos de cuatro determinaciones diarias.

\section{Nivel intermedio o normal}

En este nivel, la resistencia del hormigón se controla en forma no sistemática. Puede emplearse en obras de importancia media, teniendo en cuenta las siguientes observaciones:

- la resistencia característica de proyecto $\left(f_{c k}\right)$ no debe superar los $250 \mathrm{kp} / \mathrm{cm}^{2}$;

- el coeficiente de minoración del hormigón $\left(\gamma_{c}\right)$ puede considerarse igual a 1,50 ;

- aunque no se establece una periodicidad fija, debe procurarse que al final de la obra se hayan realizado, por lo menos, tantos ensayos como quincenas haya durado el hormigonado;

- la resistencia deducida de las probetas debe superar a la especificada. Este punto lo veremos con más detalle.

\section{Nivel superior o intenso}

En este nivel se efectúa un control sistemático de la resistencia del hormigón, a base de ensayos periódicos cuyos resultados pueden hacer variar la intensidad de muestreo. Conviene emplear este nivel de control en todas las obras de hormigón, pudiendo ser más o menos rigurosos en el tamaño de la muestra y en la frecuencia de los ensayos, en función de la importancia de la obra. Este nivel de control es de empleo obligado cuando la resistencia característica especificada o de proyecto $\left(\boldsymbol{f}_{c k}\right)$ es mayor de $250 \mathrm{kp} / \mathrm{cm}^{2}$; y también cuando se adopta para el coeficiente de minoración del hormigón $\left(\gamma_{c}\right)$ un valor inferior a 1,50 .

El procedimiento operatorio lo veremos con detalle más adelante (apartado 9) .

\section{TIPIFICACION DE HORMIGONES}

Se está tratando de llegar a una tipificación de hormigones, a escala internacional, sobre la base del valor de su resistencia característica a los 28 días de edad, expresada en $\mathrm{N} / \mathrm{mm}^{2}$ (1). La siguiente serie de valores ha sido ya aceptada por el Comité Editorial CEBCIB-FIP-RILEM:

$$
10-15-20-25-30-40-50-60 \mathrm{~N} / \mathrm{mm}^{2} \text {. }
$$

Hay que hacer notar que las formas de pro. betas no son idénticas en todos los países, subsistiendo la cilíndrica $15 \times 30$ y la cúbica $15 \times 15 \times 15$. Además, las condiciones de curado son también diferentes, lo que dificulta las equivalencias.

En España, la Instrucción EH 73 ha tipificado la siguiente serie:

H-50; H-100; H-125; H-150; H-175; H-200; $\mathrm{H}-250 ; \mathrm{H}-300 ; \mathrm{H}-350 ; \mathrm{H}-400 ; \mathrm{H}-500$,

en la cual los números indican la resistencia característica en probeta cilíndrica, expresada en $\mathrm{kp} / \mathrm{cm}^{2}$. Los tipos $\mathrm{H}-50$ y $\mathrm{H}-100$ sólo se utilizan para hormigón en masa; los $\mathrm{H}-125$ a $\mathrm{H}-250$ se emplean, generalmente, en edificación; y los restantes, en ingeniería civil y prefabricación.

(1) Recordamos que $1 \mathrm{~N} / \mathrm{mm}^{2}$ es aproximadamente igual a $10 \mathrm{kp} / \mathrm{cm}^{2}$. 


JUICIO BASADO EN $\left\{\begin{array}{l}\begin{array}{l}\text { las proporciones de la mezcla } \\ \text { (dosificación prescrita) } \\ \left(f_{c k}<30 \mathrm{~N} / \mathrm{mm}^{2}\right)\end{array} \\ \begin{array}{l}\text { la resistencia especificada } \\ \text { (dosificación proyectada) }\end{array}\end{array}\left\{\begin{array}{l}\text { Nivel 1A: No se rompen probetas } \\ \text { Nivel 1B: Se rompen algunas, para información } \\ \text { Nivel 2A: Intensidad media de ensayos de rotura }\end{array}\right.\right.$

Fig. 2

\section{FORMAS DE ESPECIFICAR EL HORMIGON}

Según los casos y países, el hormigón se especifica por resistencia o por dosificación.

En el primer caso, llamado de dosificación proyectada, se especifica el valor de $\mathbf{f}_{c k}$ y alguna otra limitación, tal como el tamaño máximo del árido y el contenido mínimo en cemento. Este es el caso de la Instrucción española EH 73.

En el segundo caso, llamado de dosificación prescrita, se especifica una determinada dosificación de las que hay tipificadas en el Reglamento Nacional correspondiente. Esta variante se admite en muchos países (Holanda, Inglaterra, Alemania), especialmente en aquéllos en que las características de las materias primas son bastante homogéneas en todo el país, sea globalmente, sea por regiones amplias. Pero esto no sucede en España, especialmente con los áridos.

Los niveles de control vienen definidos en el cuadro de la figura 2.

Como puede apreciarse, el caso español corresponde a:

$\begin{array}{lllll}\text { Nivel inferior ............. } & \ldots & \text { Nivel 1A } \\ \text { Nivel intermedio } & \ldots & \ldots & \ldots & \text { Nivel 2A } \\ \text { Nivel superior } & \ldots & \ldots & \ldots & \text { Nivel 2B }\end{array}$

Cuando se utilizan dosificaciones prescritas, tomadas de las Normas, debe elegirse la que corresponde a una resistencia adecuadamente superior a la especificada para tener un margen de reserva. Este margen debe ser del orden de $10 \mathrm{~N} / \mathrm{mm}^{2}$ a $15 \mathrm{~N} / \mathrm{mm}^{2}$, dadas las incertidumbres asociadas al empleo de dosificaciones prescritas.

Cuando se utilizan dosificaciones proyectadas, el Constructor establece la dosificación y la somete a aprobación. A efectos de diseño de la mezcla, para prever el oportuno margen de resistencia, se considerará como desviación típica (s) la dada por la figura 3 .
Como puede verse en la figura:

a) Sino hay datos previos o se dispone de menos de 30 resultados, se tomará el valor de $\mathbf{s}$ de la curva A.

b) Si hay entre 30 y 100 resultados, se tomará $\mathbf{s}$ del cálculo, pero no menor que el valor dado por la curva B.

c) Si hay más de 100 resultados, se tomará s del cálculo, pero no menor que el valor dado por la curva C.

Conviene destacar que, como indica la figura 3, se supone constante la desviación típica (parámetro dimensional) y no el coeficiente de variación (parámetro adimensional) como hasta ahora se venía haciendo (1). Este enfoque se ajusta más a la realidad, según las últimas investigaciones sobre el tema.

\section{ESTIMADORES, CURVAS DE EFICACIA} $Y$ FUNCIONES DE ACEPTACION

Como es sabido un estimador es una fórmula matemática que, particularizada para los va-

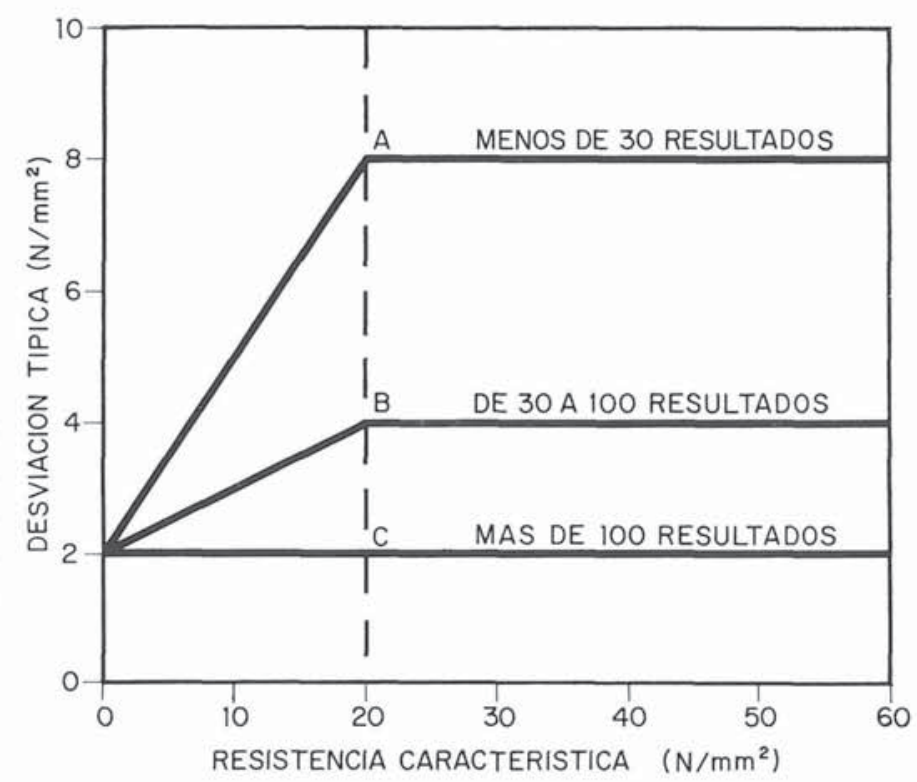

Fig. 3

(1) El Código $\mathrm{ACl}$ y la Instrucción EH-73 suponen constante el coeficiente de variación. 
lores obtenidos en el ensayo de las probetas, proporciona el valor de la resistencia característica estimada $\left(\mathbf{f}_{\text {est }}\right)$.

Como el valor de la resistencia característica está asociado a un nivel de confianza del $95 \%$, es posible definir diversos estimadores de la misma haciendo uso de los principios de la Estadística. Se trata, en definitiva, de estimar, en una distribución que se supone normal (gaussiana), aquel valor que deja a su izquierda un área del $5 \%$ del área total bajo la curva.

El estimador que fija la Instrucción española EH 73 responde a la fórmula general:

$$
\mathbf{f}_{\text {est }}=2 \frac{\mathbf{x}_{1}+\mathbf{x}_{2}+\ldots+\mathbf{x}_{n-1}}{\mathbf{n}-1}-\mathbf{x}_{n} \nless \beta \cdot \mathbf{x}_{1} ;
$$

donde:

$$
\mathbf{x}_{1} \gtrless \mathbf{x}_{2} \gtrless \ldots \gtrless \mathbf{x}_{2 n}
$$

son los resultados obtenidos al ensayar un conjunto par de $2 \mathbf{n}$ probetas. Al utilizar sólo la mitad más baja de resultados, este estimador no penaliza la dispersión por resultados elevados, con lo que no se cometen grandes errores en los casos en que la muestra proviene de dos poblaciones diferentes de probetas (distribución no gaussiana). Este caso puede presentarse por cambio en la partida de cemento, siendo la segunda más resistente que la primera.

El límite inferior $\beta \cdot \mathbf{x}_{1}$ sólo es operante en casos de muestras particularmente distorsionadas, para los cuales la expresión polinómica daría un resultado aberrante. El coeficiente $\beta$ varía con el tamaño de la muestra y el grado de uniformidad del hormigón, de acuerdo con los valores de la tabla adjunta:

VALORES DEL COEFICIENTE $\beta$

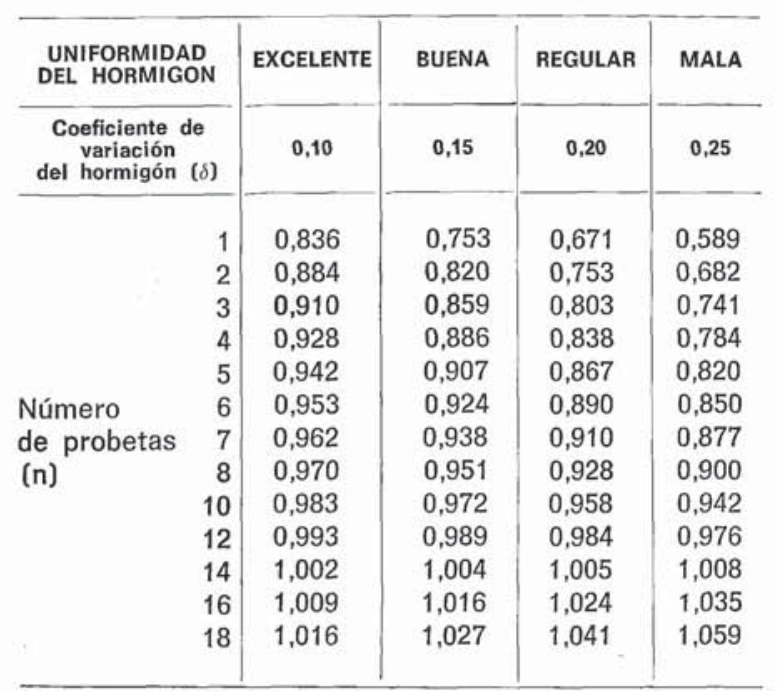

Las columnas retenidas por la EH 73 son las de $\beta=0,20$ para el caso de hormigones fabricados en hormigonera y $\beta=0,10$ para hormigones fabricados en central permanente.

El estimador $\mathbf{f}_{\text {est }}$ así definido es centrado con respecto a la resistencia característica real $\left(\mathfrak{f}_{c k, \text { real }}\right)$ del hormigón -imposible de conocer en la práctica-, lo que significa que el valor obtenido al aplicar la expresión

$$
\mathbf{f}_{\text {est }}=2 \frac{\mathbf{x}_{1}+\mathbf{x}_{2}+\ldots+\mathbf{x}_{n-1}}{\mathbf{n}-1}-\mathbf{x}_{n},
$$

a un conjunto de $2 \mathbf{n}$ probetas tiene una probabilidad de 0,5 de ser mayor que $\mathbf{f}_{c k \text {, real }}$ (y por tanto, la misma probabilidad de ser menor).

De lo anterior se deduce que si el hormigón puesto en obra tiene una resistencia estrictamente igual a la especificada $\left(\mathbf{f}_{c k}\right.$, real $\left.=\mathbf{f}_{c k}\right)$, la probabilidad de que resulte $\mathbf{f}_{e s t}>\mathbf{f}_{c k}$ es la misma que la de que resulte $\mathbf{f}_{e s t}<\mathbf{f}_{c k}$. Esta circunstancia, justa pero severa, se compensa con el hecho de colocar la aceptación automática al nivel $0,9 \mathbf{f}_{c k}$ en vez de al nivel $\mathbf{f}_{c k}$ (ver apartado 9).

En cambio, a poco que se mejore el hormigón $\left(\mathbf{f}_{c k \text {, real }}>\mathbf{f}_{c k}\right.$ ), la probabilidad de que sea $\mathbf{f}_{e s t}>\mathbf{f}_{c k}$ crece rápidamente; y viceversa cuando el hormigón empeora. Esto se ilustra en las figuras 4 y 5 .

Las curvas de eficacia de un estimador cuantifican las probabilidades mencionadas, estableciendo una relación entre el cociente $\mathbf{f}_{c k \text {, real }} / \mathbf{f}_{c k}$ y la probabilidad de que resulte $\mathbf{f}_{\text {est }}>\mathbf{f}_{c k}$. Dicha relación es distinta para cada tamaño de muestra (n) y cada coeficiente de variación $(\delta)$ del hormigón (figs. 6 y 7 ).

Las normas de cada país pueden imponer el empleo de otros estimadores, los cuales tendrán sus curvas de eficacia correspondientes. La comparación entre estas curvas permite conocer si los criterios de aceptación y rechazo de una determinada norma son más o menos severos que los de otra (1).

Así, por ejemplo, las normas españolas de barras corrugadas UNE 36088 utilizan como estimador del valor característico la media aritmética del octavo más bajo de valores, que también corresponde al cuantil $5 \%$.

(1) Si se hace este tipo de comparación, no debe olvidarse todo lo dicho en el apartado 3. 

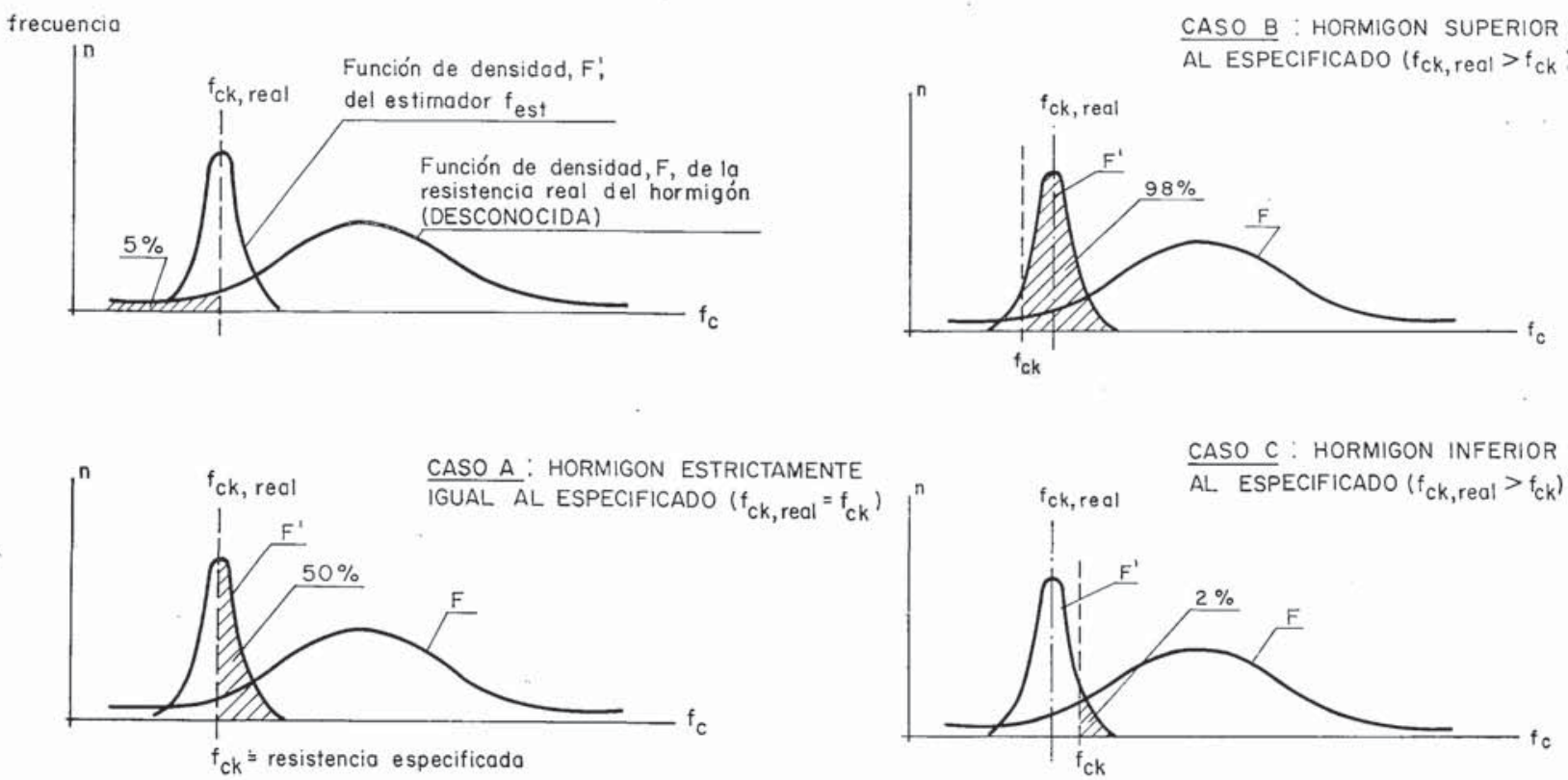

Figs. 4 y 5
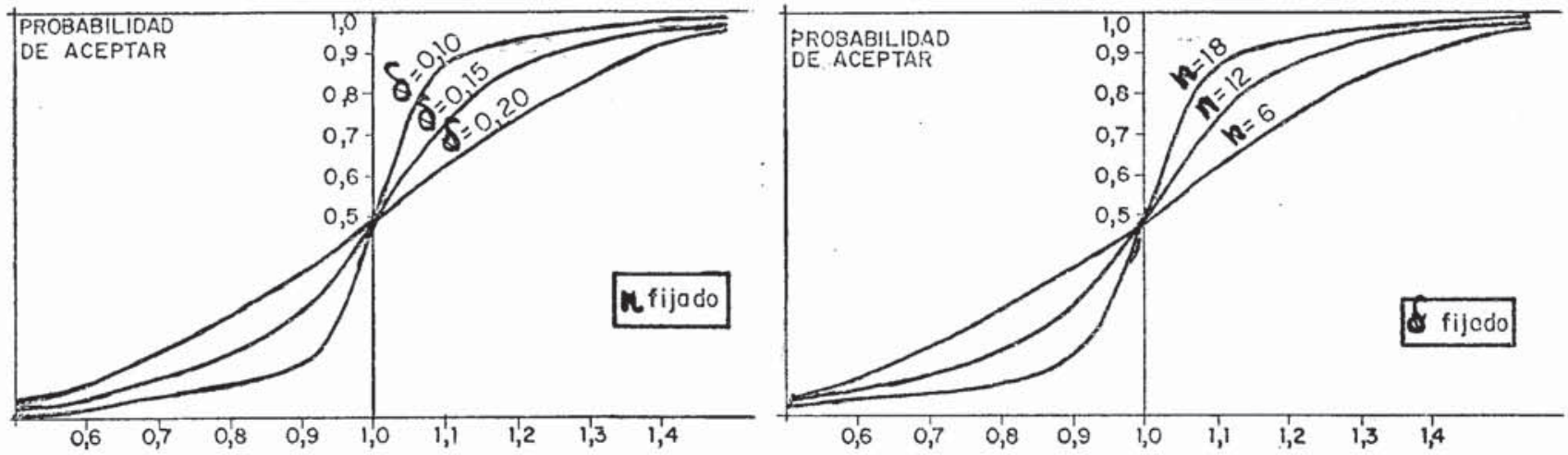

Fig. 6

Las normas del Comité Europeo del Hormigón presentan el estimador en su forma clásica:

$$
\mathbf{f}_{c k}=\mathbf{f}_{c m}(1-\lambda \cdot \delta),
$$

donde:

$$
\begin{aligned}
\mathbf{f}_{c m}= & \text { resistencia media de una muestra } \\
& \text { de } \mathbf{n} \text { probetas. } \\
\delta= & \text { coeficiente de variación. } \\
\lambda= & \text { coeficiente cuyo valor depende del } \\
& \text { número } \mathbf{n} \text { de probetas. }
\end{aligned}
$$

Pero este tipo de estimadores, estadísticamente correctos, suelen resultar severos en la práctica, sobre todo en hormigones de resistencia media o baja, cuya distribución de resistencia puede diferir de la gaussiana; y además, conducen a valores excesivamente bajos de $\mathbf{f}_{c k}$ cuando por azar, descuido o desconocimiento, se han mezclado en la muestra dos poblaciones diferentes.
En la actualidad continúan los estudios sobre este tema, con vistas a una normalización internacional de criterios de control de calidad. Son de destacar al respecto los trabajos de Rüsch y Rackwitz (Munich), Hardman y Teychenne (Londres), y Wiebenga (Delf). A título orientativo, daremos a continuación las ideas que parece gozan de más aceptación a nivel europeo hoy.

Las preferencias se orientan hacia estimadores clásicos del tipo:

$$
\mathbf{f}_{\text {est }}=\mathbf{f}_{m}-\lambda \cdot \mathbf{s},
$$

donde:

$$
\begin{aligned}
\mathbf{f}_{m}= & \text { resistencia media. } \\
\mathbf{s}= & \text { desviación típica. } \\
\lambda= & \text { coeficiente en función del número } \\
& \text { de probetas } \mathbf{n} \text { (ver fig. } 8 \text { ). }
\end{aligned}
$$




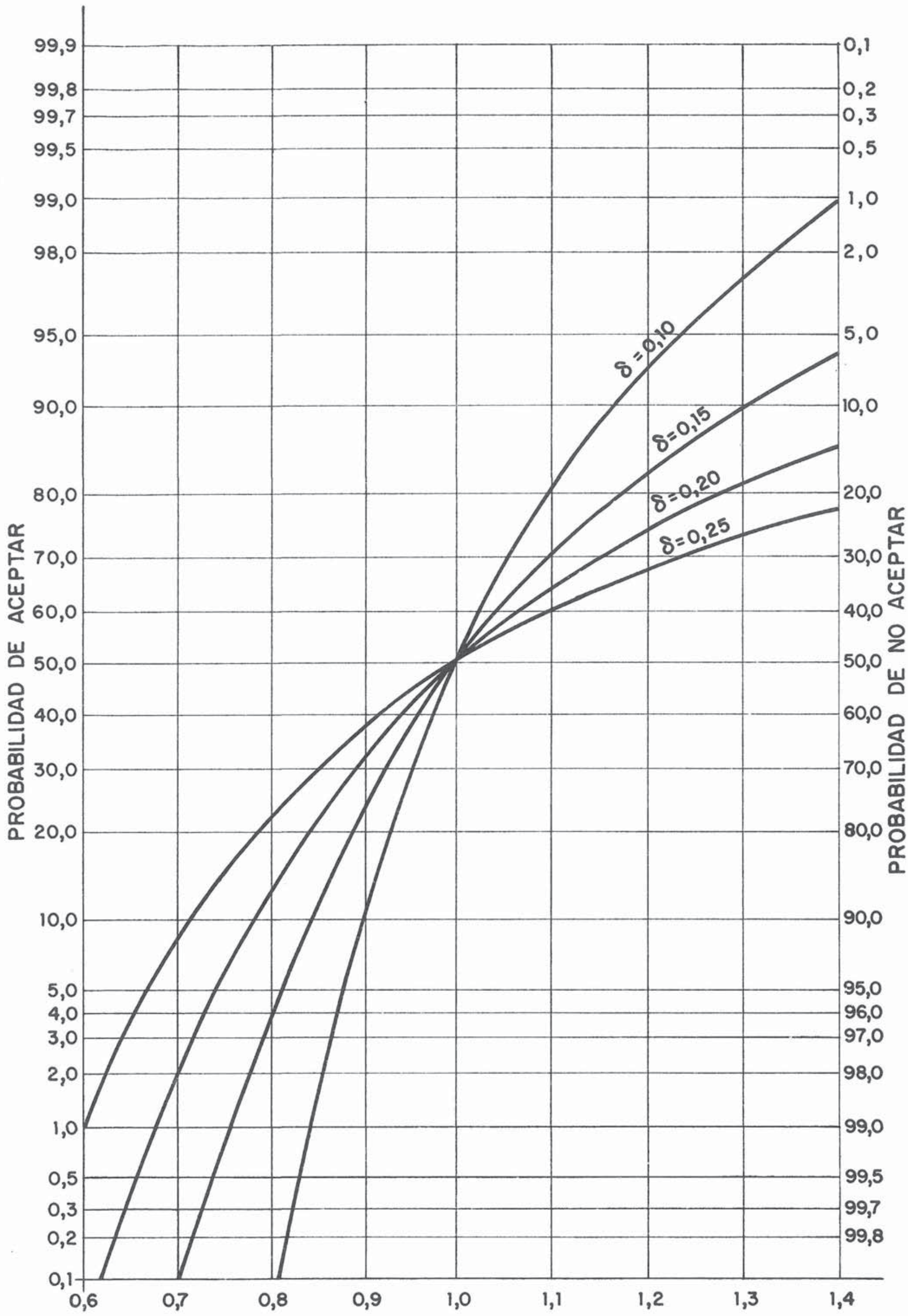

Fig. 7. Curvas de eficacia del estimador español de la EH-73 para $n=6$ probetas. 


\begin{tabular}{|c|c|c|c|c|c|c|c|c|c|}
\hline \multirow{2}{*}{$\begin{array}{l}\text { Desviación típica conocida } \\
\text { o especificada }\end{array}$} & $\mathbf{n}$ & 3 & 4 & 5 & 6 & 7 & 8 & 9 & 10 \\
\hline & $\lambda$ & 1,71 & 1,56 & 1,45 & 1,37 & 1,31 & 1,27 & 1,22 & 1,18 \\
\hline \multirow{2}{*}{$\begin{array}{l}\text { Desviación típica } \\
\text { desconocida }\end{array}$} & $\mathbf{n}$ & 4 & 6 & 8 & 10 & 12 & 14 & 20 & \\
\hline & $\lambda$ & 2,92 & 2,24 & 1,63 & 1,38 & 1,27 & 1,22 & 1,1 & \\
\hline
\end{tabular}

$\mathbf{n}=$ número mínimo de probetas necesarias para tomar decisión.

Fig. 8

Puede verse que es necesario duplicar el tamaño de muestra (aproximadamente) si la s es desconocida. Para producción estable puede bastar con $\mathbf{n}=30$ a $\mathbf{n}=40$ al objeto de calcular $\mathbf{n}$.

A efectos prácticos, se recomienda utilizar como estimador el correspondiente a $\lambda=1,4$, como indica la figura 9 .

$$
\begin{gathered}
f_{c, e s t}=f_{c m}-1,4 s \\
s=\text { DESVIACION TIPICA } \\
n=6 \text { PARA s PREFIJADA } \\
n=10 \text { PARA s DESCONOCIDA }
\end{gathered}
$$

Fig. 9

La comparación de este criterio con el criterio español puede verse en las curvas de eficacia correspondientes. En la figura $10 \mathrm{se}$ muestra otra forma de representar curvas de eficacia, diferente de la de las figuras 6 y 7 . En ordenadas seguimos teniendo la probabilidad de aceptar, pero en abscisas representamos ahora la proporción de defectuosos. Quiere esto decir que la ordenada $5 \%$ corresponde a la resistencia característica real, o sea, el valor del cociente $\mathbf{f}_{c k \text {, real }} / \mathbf{f}_{c k}$ de la figura 7. Con esta forma de representación, en papel logarítmico, las curvas de eficacia resultan rectas.

Las dos zonas rayadas de la figura 10 definen dos fronteras establecidas por el Comité Editorial CEB-CIB-FIP-RILEM. Cualquier estimador o función de aceptación debe mantenerse en la zona intermedia, sin cortar a las fronteras, como sucede con los dos representados (propuestas alemana y española).
El gráfico de la figura 10 indica que, por ejemplo, un hormigón con un $30 \%$ de defectuosos no debe tener una probabilidad de aceptación mayor del $2 \%$.

Como se ve, el estimador español es más severo, ya que un hormigón crítico $(5 \%$ de defectuosos) es aceptado por el estimador alemán en un $75 \%$ de los casos, y sólo en un $50 \%$ de los casos por el español. Esta circunstancia queda compensada por el hecho de que los criterios de aceptación-rechazo son más tolerantes en el caso español.

Ahora bien, no todas las Normas emplean estimadores de $\mathbf{f}_{c k}$. Algunas Normas utilizan, en su lugar, funciones de aceptación. Así, por ejemplo, el Código inglés CP 110 , recientemente publicado, establece el siguiente criterio:

- para $\mathbf{f}_{c k}<225 \mathrm{kp} / \mathrm{cm}^{2}$, debe ser:

$$
\left\{\begin{array}{l}
\mathbf{f}_{m}>\mathbf{f}_{c k}+1 / 3 \mathbf{f}_{c k} \\
\mathbf{f}_{i}>0,85 \mathbf{f}_{c k} ;
\end{array}\right.
$$

- para $\mathbf{f}_{c k}>225 \mathrm{kp} / \mathrm{cm}^{2}$, debe ser:

donde:

$$
\left\{\begin{array}{l}
\mathbf{f}_{m}>\mathbf{f}_{c k}+75 \mathrm{kp} / \mathrm{cm}^{2} ; \\
\mathbf{f}_{i}>0,85 \mathbf{f}_{c k} ;
\end{array}\right.
$$

$$
\begin{aligned}
\mathbf{f}_{m} & =\text { media de cuatro probetas; } \\
\mathbf{f}_{i} & =\text { valor más bajo de ellas. }
\end{aligned}
$$

El criterio se aplica a cada nueva probeta extraída, que se liga con las tres precedentes.

Otro ejemplo lo constituye el Código Americano del A.C.I., que exige que la media de tres probetas consecutivas supere la resistencia 


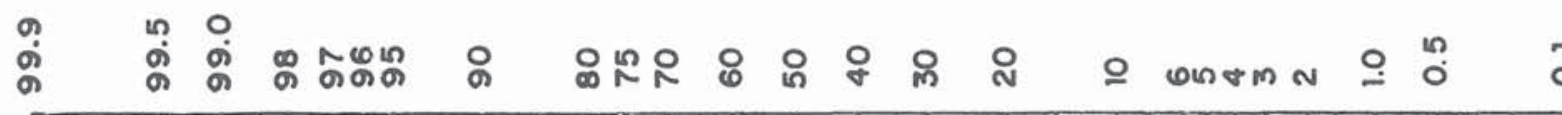

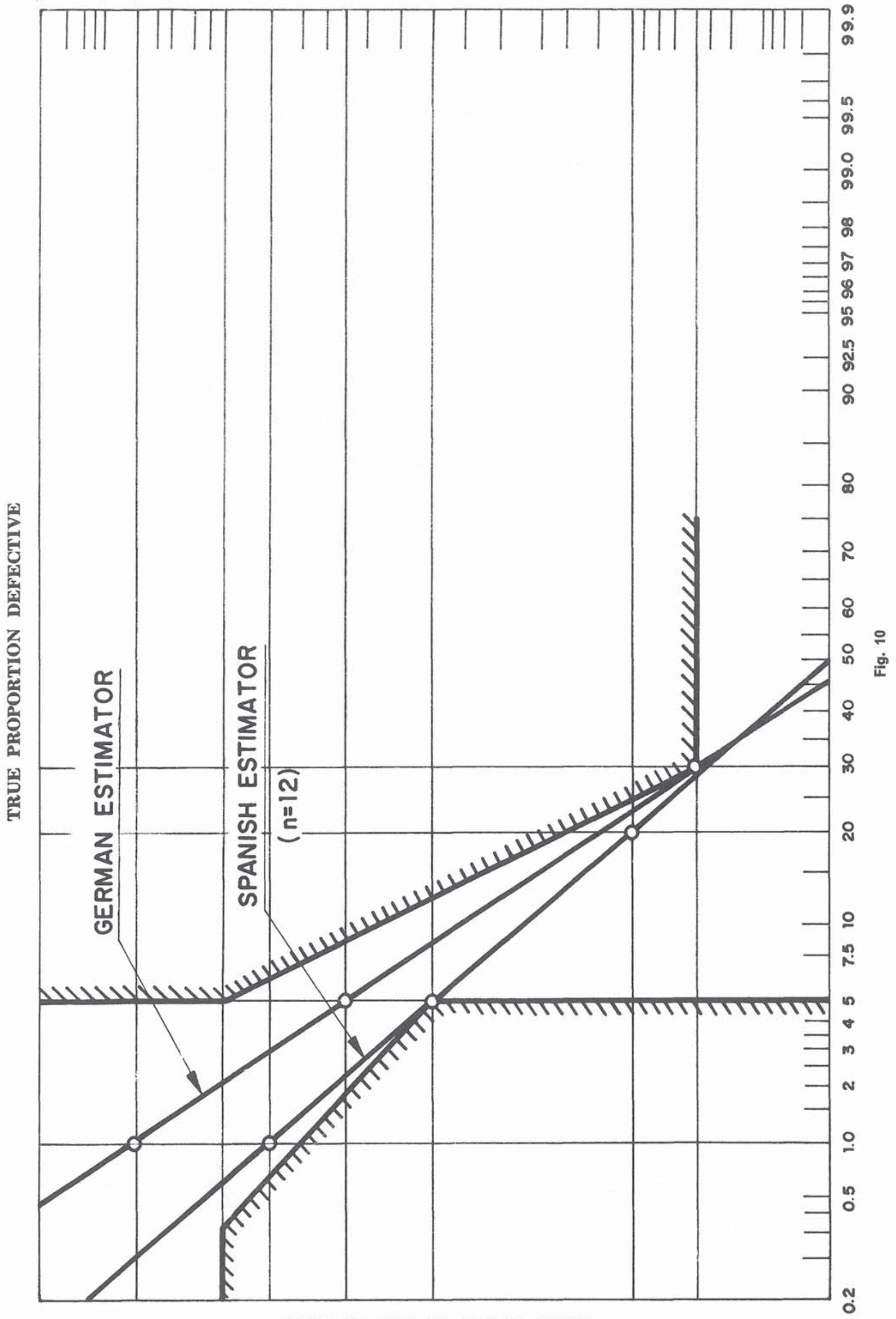

(c) Consejo Superior de Investigaciones CiBABILITY OF ACCEPTANCE 
especificada, y que la probeta más baja no se separe de ella por abajo en más de $35 \mathrm{kp} / \mathrm{cm}^{2}$. Este criterio es muy tolerante, como puede apreciarse. Pero ello no significa necesariamente una menor seguridad, ya que habría que comparar otras cosas (valores de $\gamma$, fórmulas de cálculo, etc.). Comparación que no es inmediata, porque la filosofía del Código A.C.I. no es exactamente la misma que en Europa (método de los Estados Límites).

\section{EXTENSION DEL LOTE $Y$ CONSTITUCION DE LA MUESTRA}

La extensión de cada lote de control debe venir fijada en el Pliego de Prescripciones Particulares o, en su defecto, ser establecida por el Director de Obra. Como es evidente, cuanto mayor es el lote se producen menos gastos de ensayo, pero aumenta el riesgo de tomar decisiones incorrectas.

En general, se deben agrupar en un solo lote aquellos elementos afines que se hormigonan de forma continuada en el tiempo. A título orientativo, la Instrucción española EH 73 incluye los cuadros de la figura 11 , correspondientes a nivel normal y nivel intenso de control.

El tamaño normal de la muestra es de 6 probetas. En casos de muestreo intenso suelen utilizarse $12 \mathrm{y}$, excepcionalmente, 18 probetas por lote.

Las probetas que se toman para constituir la muestra deben proceder, en principio, de diferentes amasadas, con objeto de recorrer el máximo número posible de éstas. No obstante, cuando las amasadas son de gran volumen se suele admitir la toma de más de una probeta de una misma amasada, sin rebasar el máximo de tres. De esta forma, siempre estarán representadas, en la muestra, al menos dos amasadas.

Lo dicho se refiere al caso de control efectuado por personas u organizaciones ajenas al constructor, que no están en obra de forma continuada. Si se trata de un control efectuado por el propio constructor (1), la información puede ser más continua, debiendo entonces tomarse, al menos, una probeta en cada día de hormigonado y no menos de una probeta por cada:

- $10 \mathrm{~m}^{3}$ ó 10 amasadas, en los casos de muestreo intenso;

- $20 \mathrm{~m}^{3}$ ó 20 amasadas en los casos de muestreo normal;

- $50 \mathrm{~m}^{3}$ ó 50 amasadas, en los casos de muestreo reducido;

siendo operante el menor de los dos valores indicados en cada caso.

\section{CRITERIOS DE ACEPTACION O RECHAZO.}

Las distintas alternativas que pueden presentarse están recogidas en los cuadros adjuntos, que han sido aprobados por el Comité Editorial CEB-CIB-FIP-RILEM. Como se ve en ellos, el rechazo sólo se produce después de haber agotado todas las posibilidades que conducirían a una aceptación.

(1) De nuevo aparece aquí la diferencia entre control de aceptación y control de producción.

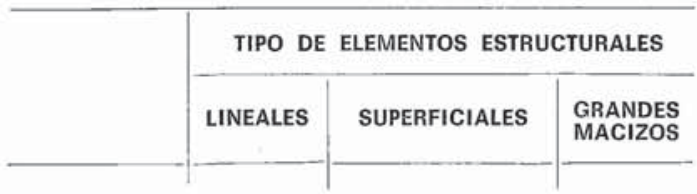

\begin{tabular}{lccc} 
Volumen & $100 \mathrm{~m}^{3}$ & $200 \mathrm{~m}^{3}$ & $500 \mathrm{~m}^{3}$ \\
\hline Superficie & $500 \mathrm{~m}^{2}$ & $500 \mathrm{~m}^{2}$ &
\end{tabular}

NIVEL NORMAL

NIVEL INTENSO

\begin{tabular}{|c|c|c|c|}
\hline & \multicolumn{3}{|c|}{ TIPO DE ELEMENTOS ESTRUCTURALES } \\
\hline & LINEALES & SUPERFICIALES & $\begin{array}{l}\text { GRANDES } \\
\text { MACIZOS }\end{array}$ \\
\hline Por volumen & $100 \mathrm{~m}^{3}$ & $200 \mathrm{~m}^{3}$ & $500 \mathrm{~m}^{3}$ \\
\hline Por superficie & $400 \mathrm{~m}^{2}$ & $400 \mathrm{~m}^{2}$ & - \\
\hline $\begin{array}{l}\text { Por tiempo (hor- } \\
\text { migón coloca- } \\
\text { do en) }\end{array}$ & 2 semanas & 2 semanas & 1 semana \\
\hline Por planta & 1 & 1 & - \\
\hline
\end{tabular}

Fig. 11 
En España, esta filosofía general se aplica de forma detallada en la manera que ilustra la figura 12. De acuerdo con la tabla que en ella aparece, cuando resulta $\mathbf{f}_{e s t}>\mathbf{f}_{c k}$ se acepta automáticamente el hormigón. Cuando $\mathbf{f}_{e s t}<\mathbf{f}_{c k}$, pero es $\mathbf{f}_{\text {est }}>0,9 \mathbf{f}_{c k}$, el hormigón puede también aceptarse, ya que el estimador que define $\mathbf{f}_{\text {est }}$ es más bien severo; pero procede en tal caso imponer una penalización (que puede ser de tipo económico y proporcional al descenso de resistencia, si así lo impone el Pliego de Prescripciones Particulares) y, sobre todo, procede aumentar al doble el tamaño de la muestra (en el caso de que ésta fuera de 6 probetas) con objeto de estimar, en lo sucesivo, la resistencia real del hormigón de forma más precisa.

Si el descenso de resistencia fuese mayor del $10 \% \quad$ (f $\boldsymbol{f}_{\text {est }}<$ $<0,9 \mathbf{f}_{c k}$ ) es obligado efectuar un estudio particular para cuantificar el reflejo que dicho descenso puede tener en la seguridad de los elementos afectados.

Así, por ejemplo, si se trata de soportes el problema tendrá más importancia que si se trata de vigas. Después comentaremos más detenidamente este tema.

En cualquier caso, siempre que resulte $\mathbf{f}_{e s t}<\mathbf{f}_{c k}$ es conveniente proceder a la extracción de probetas-testigo, en número no inferior a seis (1), con objeto de tener una estimación más precisa de la resistencia del hormigón en entredicho. Si se hace así, la base de juicio se traslada automáticamente de las probetas enmoldadas a las testigo, debiendo aplicarse el criterio de aceptación o rechazo que se indica en el apartado 10.

(1) Petersons recomienda un mínimo de $10 \mathrm{y}$, si se trata de diversos elementos estructurales, un minimo de 3 por elemento. cuadro original

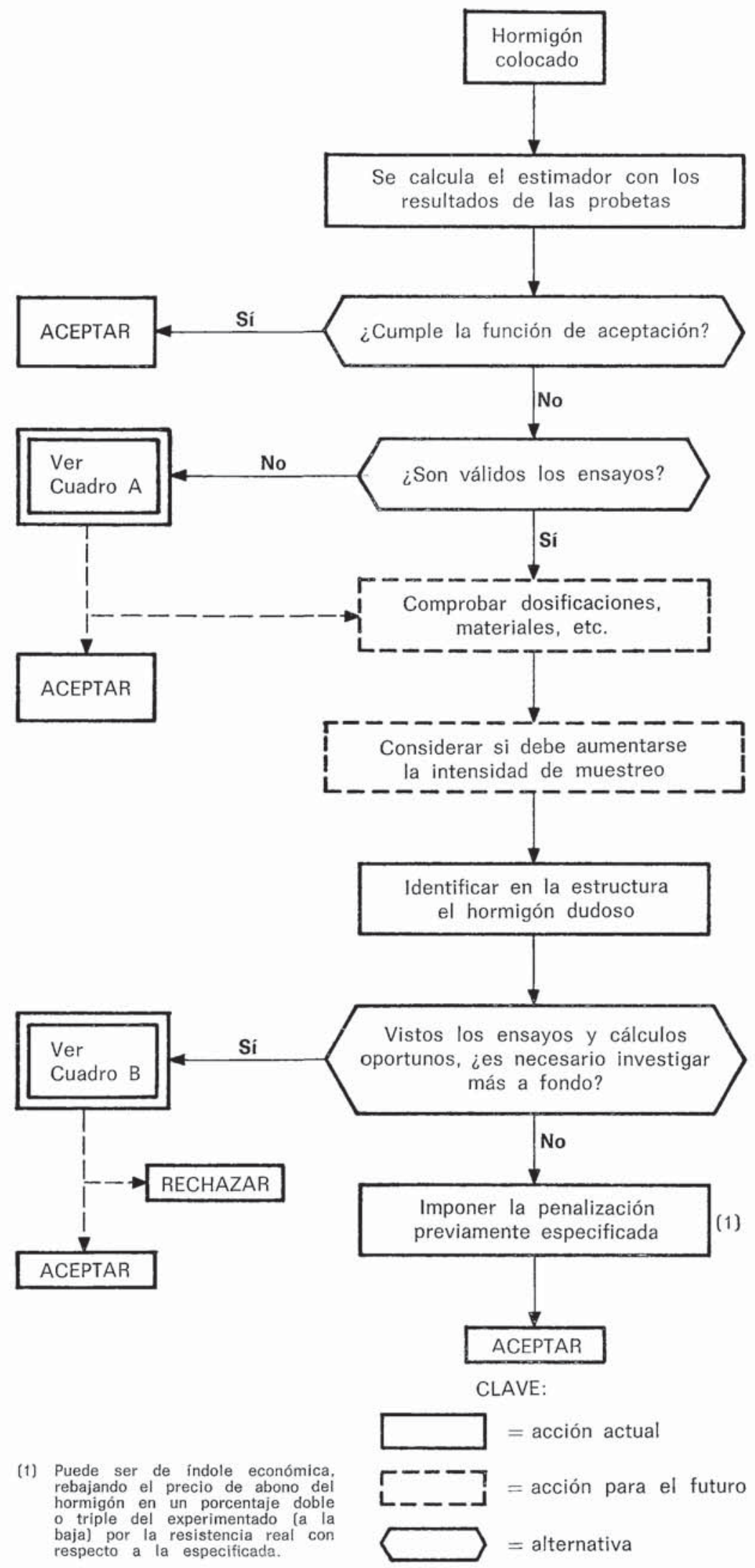



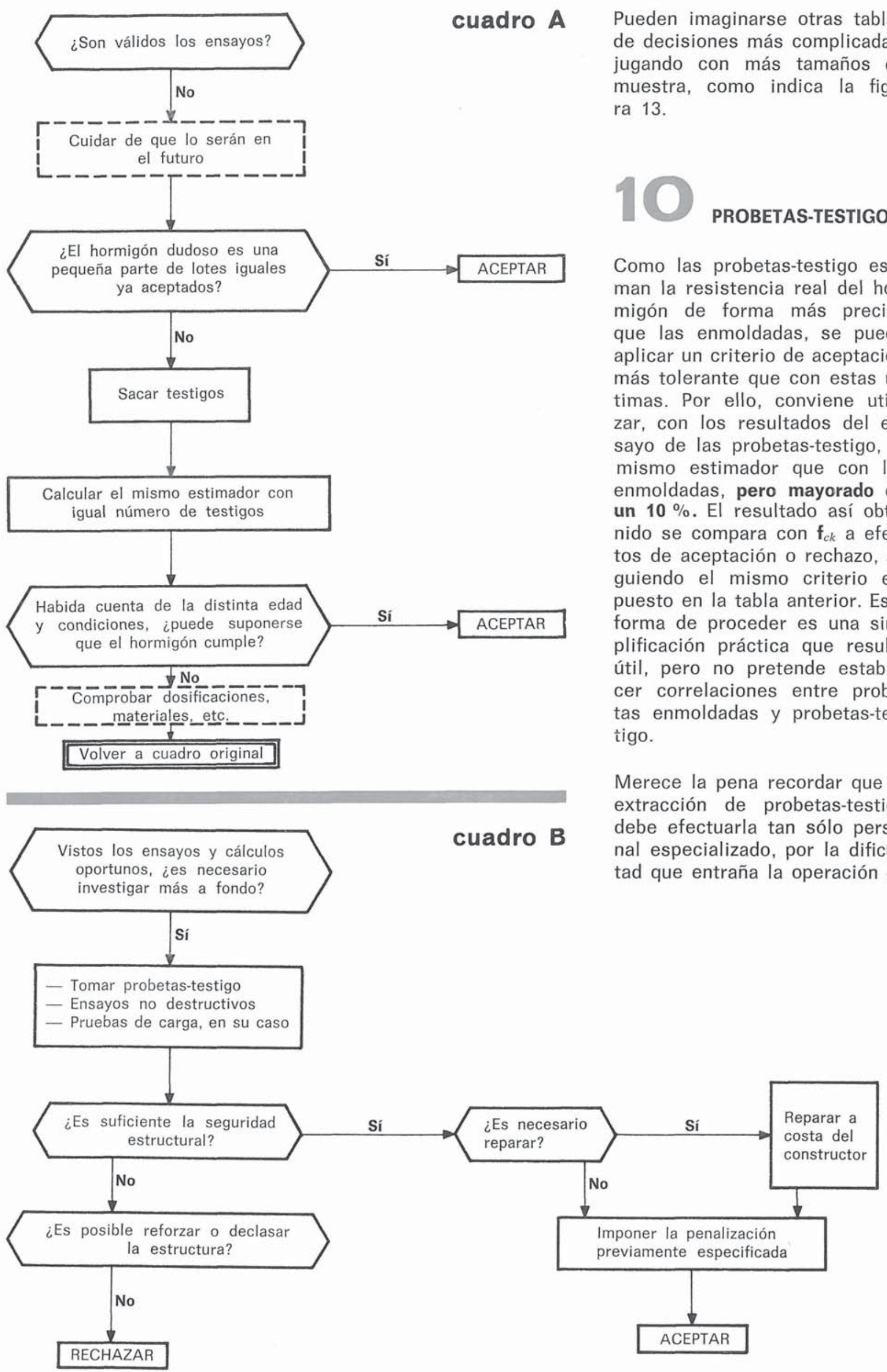

Pueden imaginarse otras tablas de decisiones más complicadas, jugando con más tamaños de muestra, como indica la figura 13.

\section{PROBETAS-TESTIGO}

Como las probetas-testigo estiman la resistencia real del hormigón de forma más precisa que las enmoldadas, se puede aplicar un criterio de aceptación más tolerante que con estas últimas. Por ello, conviene utilizar, con los resultados del ensayo de las probetas-testigo, el mismo estimador que con las enmoldadas, pero mayorado en un $10 \%$. El resultado así obtenido se compara con $\mathbf{f}_{c k}$ a efectos de aceptación o rechazo, siguiendo el mismo criterio expuesto en la tabla anterior. Esta forma de proceder es una simplificación práctica que resulta útil, pero no pretende establecer correlaciones entre probetas enmoldadas y probetas-testigo.

Merece la pena recordar que la extracción de probetas-testigo debe efectuarla tan sólo personal especializado, por la dificulad que entraña la operación de 
TABLA SIMPLIFICADA DE DECISIONES

\begin{tabular}{|c|c|c|c|}
\hline \multirow{2}{*}{$\begin{array}{l}\text { Número } \\
\text { de probetas }\end{array}$} & \multirow[b]{2}{*}{ ALTERNATIVAS } & \multicolumn{2}{|c|}{ DECISIONES } \\
\hline & & $\begin{array}{l}\text { Aceptación } \\
\text { automática }\end{array}$ & $\begin{array}{l}\text { Pasar a número de } \\
\text { probetas igual a: }\end{array}$ \\
\hline \multirow{3}{*}{6} & $\mathbf{f}_{e s t}>\mathbf{f}_{c k}$ & Sí & 6 \\
\hline & $\mathbf{f}_{c k}>\mathbf{f}_{e s t}>0,9 \mathbf{f}_{c k}$ & $\begin{array}{c}\text { Sí } \\
\text { con penalización }\end{array}$ & 12 \\
\hline & $\mathbf{f}_{e s t}<0,9 \mathbf{f}_{c k}$ & No & 12 \\
\hline \multirow{3}{*}{12} & $\mathbf{f}_{e s t}>\mathbf{f}_{c k}$ & Sí & 6 \\
\hline & $\mathbf{f}_{c k}>\mathbf{f}_{e s t}>0,9 \mathbf{f}_{c k}$ & $\begin{array}{c}\text { Sí } \\
\text { con penalización }\end{array}$ & 12 \\
\hline & $\mathbf{f}_{e s t}<0,9 \mathbf{f}_{c k}$ & No & 12 \\
\hline
\end{tabular}

Fig. 12

forma fiable. En particular debe tenerse en cuenta que:

- No existe una correlación exacta entre la resistencia de probetas-testigo y la de probetas enmoldadas, dando las primeras casi siempre resultados inferiores a las segundas, en proporción variable entre el 5 y el $10 \%$. En general, la diferencia es tanto mayor cuanto más resistente es el hormigón.

- La resistencia de las probetas-testigo aumenta, para un mismo elemento (soporte, muro, viga o losa), con la profundidad del punto de extracción, respecto a la superficie más alta del elemento.

- Debe distinguirse claramente qué es lo que se persigue al extraer probetas-testigo. Si se busca saber si se ha cumplido o no un contrato, las probetas-testigo no sirven, ya que el contrato se establece sobre las enmoldadas. Si se busca conocer el margen de seguridad probable de la obra ejecutada, hay que proceder a un estudio especial en cada caso (ver apartado siguiente). Pero, en estricta doctrina, no debe ligarse el concepto de "resistencia característica" a los resultados de las probetas-testigo.

\section{1 \\ REPERCUSIONES EN LA SEGURIDAD DE LOS ELEMENTOS}

Cuando el control de calidad detecta, en un determinado elemento, una desviación entre la obra ejecutada y la proyectada, hay que de- terminar la influencia que dicha desviación tiene en la seguridad del elemento.

Las desviaciones pueden presentarse en las resistencias de los materiales (hormigón y acero) o en las dimensiones de las secciones (canto útil, anchura, etc.).

El caso más frecuente es aquél en que el hormigón ha experimentado un descenso de resistencia frente al valor especificado. Este es el caso que desarrollamos a continuación. Para los casos restantes se operaría en forma análoga.

Sea $\mathbf{f}_{k c}$ la resistencia especificada y $\mathbf{f}_{e s t}$ la realmente obtenida, siendo $\mathbf{f}_{s t}<\mathbf{f}_{c k}$. Un análisis riguroso del problema, planteado sobre bases estrictamente probabilistas, resulta hoy prácticamente inabordable por la complejidad del mismo (1). Pero pueden indicarse algunos criterios sencillos que permiten estimar el nivel de seguridad que se busca:

1. Se determina el coeficiente de seguridad real $\left(\gamma_{1}\right)$ que correspondería al elemento en el supuesto de que el hormigón tuviese una resistencia característica igual a la especificada $\left(\mathbf{f}_{c k}\right)$. Para ello, se rehace el cálculo de proyecto, operando ahora con una resistencia de cálculo igual a $\mathbf{f}_{c k}$, es decir, admitiendo un coeficiente de minoración $\left(\gamma_{c}\right)=1$.

(1) Ferry Borges, entre otros, se ha ocupado del estudio teórico de este tema con gran generalidad. 
2. Se determina el coeficiente de seguridad $\left(\gamma_{2}\right)$ que corresponde al elemento a partir de una resistencia característica del hormigón igual a $\boldsymbol{f}_{\text {est }}$. Es decir, se repite el cálculo anterior, cambiando $\mathbf{f}_{c k}$ por $\mathbf{f}_{\text {est }}$.

3. La comparación entre los valores de $\gamma_{1}$ y $\gamma_{2}$ permitirá tomar la decisión adecuada acerca de la conservación o demolición del elemento.

4. En los casos de elementos sometidos a una solicitación simple (flexión simple o compresión), los cálculos indicados en 1 y 2 son inmediatos, obteniéndose $\gamma$ como cociente entre la solicitación última y la característica. Si existe esfuerzo cortante, se comprobará por separado.

5. En los casos de solicitación compuesta (momento flector combinado con esfuerzo axil) la obtención de $\gamma$ no es inmediata, ya que el agotamiento puede presentarse de diferentes formas, según varien las dos solicitaciones simples. Esta situación se ilustra esquemáticamente en la figura 14.

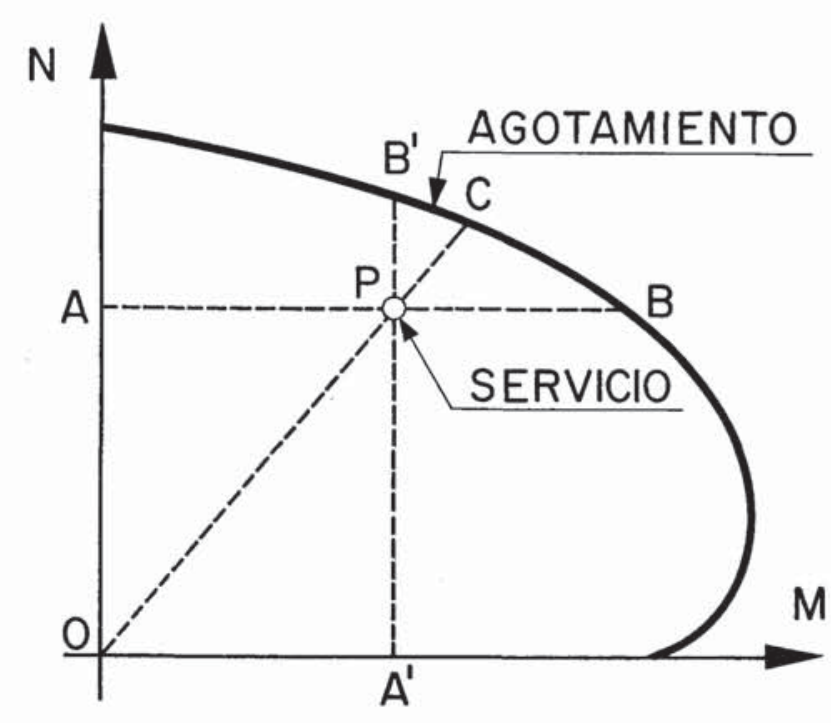

Fig. 14

tABLA DE DECISIONES CORRESPONDIENTES AL NIVEL SUPERIOR DE CONTROL

\begin{tabular}{|c|c|c|c|}
\hline \multirow{2}{*}{$\begin{array}{l}\text { Nlvel do } \\
\text { inspecclón }\end{array}$} & \multirow{2}{*}{ ALTERNATIVAS } & \multicolumn{2}{|c|}{ DECISIONES } \\
\hline & & $\begin{array}{l}\text { Aceptación } \\
\text { automática }\end{array}$ & $\begin{array}{l}\text { Pasar a número de } \\
\text { probetas igual a: }\end{array}$ \\
\hline
\end{tabular}

$\mathbf{f}_{\text {est }}>1,10 \mathbf{f}_{c k} \quad$ Sí $\quad 6$

\begin{tabular}{lll}
\hline $1,10 \mathbf{f}_{c k}>\mathbf{f}_{\text {est }}>\mathbf{f}_{c k}$ & Si & 12 \\
\hline
\end{tabular}

Reducido

\begin{tabular}{|c|c|c|c|}
\hline \multirow{2}{*}{ educido } & $\mathbf{f}_{c k}>\mathbf{f}_{e s t}>0,9 \mathbf{f}_{c k}$ & $\begin{array}{c}\text { Sí } \\
\text { con penalización }\end{array}$ & 12 \\
\hline & $\mathbf{f}_{e s t}<0,9 \mathbf{f}_{c k}$ & No & 12 \\
\hline \multirow{4}{*}{ ormal } & $\mathbf{f}_{e s t}>1,10 \mathbf{f}_{c k}$ & Sí & 6 \\
\hline & $1,10 \mathbf{f}_{c k}>\mathbf{f}_{e s t}>\mathbf{f}_{c k}$ & Sí & 12 \\
\hline & $\mathbf{f}_{c k}>\mathbf{f}_{e s t}>0,9 \mathbf{f}_{c k}$ & $\begin{array}{c}\text { Sí } \\
\text { con penalización }\end{array}$ & 18 \\
\hline & $\mathbf{f}_{e s t}<0,9 \mathbf{f}_{c k}$ & No & 18 \\
\hline \multirow{4}{*}{ guroso } & $\mathbf{f}_{e s t}>1,10 \mathbf{f}_{c k}$ & Si & 12 \\
\hline & $1,10 \mathbf{f}_{c k}>\mathbf{f}_{e s t}>\mathbf{f}_{c k}$ & Sí & 12 \\
\hline & $\mathbf{f}_{c k}>\mathbf{f}_{e s t}>0,9 \mathbf{f}_{c k}$ & $\begin{array}{c}\text { Sí } \\
\text { con penalización }\end{array}$ & 18 \\
\hline & $\mathbf{f}_{e s t}<0,9 \mathbf{f}_{c k}$ & No & 18 \\
\hline
\end{tabular}

\begin{tabular}{|c|c|c|c|}
\hline & $\mathbf{f}_{c k}>\mathbf{f}_{e s t}>0,9 \mathbf{f}_{c k}$ & $\begin{array}{c}\text { Sí } \\
\text { con penalización }\end{array}$ & 12 \\
\hline & $\mathbf{f}_{e s t}<0,9 \mathbf{f}_{c k}$ & No & 12 \\
\hline \multirow{4}{*}{ Normal } & $\mathbf{f}_{e s t}>1,10 \mathbf{f}_{c k}$ & Sí & 6 \\
\hline & $1,10 \mathbf{f}_{c k}>\mathbf{f}_{e s t}>\mathbf{f}_{c k}$ & Sí & 12 \\
\hline & $\mathbf{f}_{c k}>\mathbf{f}_{e s t}>0,9 \mathbf{f}_{c k}$ & $\begin{array}{c}\text { Sí } \\
\text { con penalización }\end{array}$ & 18 \\
\hline & $\mathbf{f}_{e s t}<0,9 \mathbf{f}_{c k}$ & No & 18 \\
\hline \multirow{4}{*}{ Riguroso } & $\mathbf{f}_{e s t}>1,10 \mathbf{f}_{c k}$ & Si & 12 \\
\hline & $1,10 \mathbf{f}_{c k}>\mathbf{f}_{e s t}>\mathbf{f}_{c k}$ & Sí & 12 \\
\hline & $\mathbf{f}_{c k}>\mathbf{f}_{e s t}>0,9 \mathbf{f}_{c k}$ & $\begin{array}{c}\text { Sí } \\
\text { con penalización }\end{array}$ & 18 \\
\hline & $\mathbf{f}_{e s t}<0,9 \mathbf{f}_{c k}$ & No & 18 \\
\hline
\end{tabular}

Fig. 13 
El proyectista debe analizar, en su caso particular, cuál es el recorrido (o los recorridos) más probable entre la situación de servicio y la de agotamiento, deduciendo de ello el valor de $\gamma$. En este análisis deben considerarse por separado las distintas acciones actuantes y su probabilidad de aumento.

Así, por ejemplo, en el caso en que el esfuerzo axil (N) esté producido por el peso propio y el momento flector (M) por el viento (acciones independientes), la seguridad a viento valdrá:

$$
\gamma=\frac{\mathbf{A B}}{\mathbf{A P}} .
$$

Por el contrario, si lo que se mantiene constante es la excentricidad con que actúa la fuerza $\mathbf{N}$ (por ejemplo, carga de un puente-grúa sobre una cartela de soporte), la seguridad valdrá:

$$
\gamma=\frac{\text { OC }}{\text { OP }} .
$$

6. Algunos autores españoles, como Villacañas y Calavera, han estudiado teóricamente la alteración de $\gamma$ en función de las diversas alteraciones en obra, estableciendo curvas de relación. En las figuras 15 y 16 se muestran dos ejemplos, entresacados de los trabajos de Calavera, que se refieren a secciones que trabajan, res. pectivamente, a flexión simple y a com. presión simple.

En conclusión, se trata de un tema en el que no pueden darse reglas precisas, siendo obligado que el técnico correspondiente adopte decisiones a su criterio y responsabilidad.

\section{BIBLIOGRAFIA}

Statistical quality control of concrete; por H. RÜSCH Informe del Laboratorio de Ensayo de Materiales de la Universidad Técnica de Munich, presentado a la conferencia sobre "Planning and Assessment of Tests", Berlín, 12 marzo 1964.

The variability of the strength of concrete and its treatment in Codes of Practice; por D. C. TEYCHENNE. Publicado en "Structural Concrete», 1966, vol. 3, enerofebrero, pp. 30-47.

Empfehlungen für die Qualitätskontrolle des Herstellers; por H. BLAUT. Informe restringido. Hay versión inglesa preparada por la B.R.S. de Londres.

Influence of variations in the strength of materials and variations in dimensions on the safety of reinforced concrete members; por J. CALAVERA. Informe presentado al CEB, Madrid, 1971

The significance of the concept of probability of failure as applied to the theory of structural safety; por H. RÜSCH y R. RACKWITZ. Publicado con ocasión del 100 aniversario de Held and Francke Bauaktiengesellschaft, Munich, 1971.

Recommendations for estimation of quality of concrete in finished structures; por N. PETERSONS. Boletín de la RILEM, núm. 24, noviembre-diciembre 1971.

Zur Streuung der Betondruckfestigkeit von Würfelproben; por R. RACKWITZ. Publicado en "Beton", 1971 (2) 53-5.

Panorama español actual del control de calidad en la construcción; por A. G. MESEGUER. "Informes de la Construcción», núm. 241, junio 1972.
Teorías probabilistas de seguridad; por J. M. ANTON Monografía núm. 306 del Instituto Eduardo Torroja, Madrid, noviembre 1972.

Recommendations for the treatment of the variation of concrete strength in Codes of Practice; por D. C. TEYCHENNE. Informe del Grupo de Trabajo núm. 1 del Comité Mixto CEB-CIB-FIP-RILEM sobre Control de Calidad. Publicado por la Building Research Station (di. fusión restringida) en noviembre 1972.

Resistencia característica y control de calidad; por un grupo de expertos de la Comisión Permanente del Hormigón. Publicado por el M.O.P., Madrid, 1972.

Statistical control in concrete structures; por R. RACKWITZ. Curso especial dictado en el Curso Internacional CEB sobre Hormigón Estructural, Lisboa, 1973. Editado por el Laboratorio Nacional de Engenharia Civil, 1973.

Quality control of concrete; por A. G. MESEGUER. Conferencia pronunciada en el Curso Internacional CEB sobre Hormigón Estructural, Lisboa, 1973. Editada por el Laboratorio Nacional de Engenharia Civil, 1973.

Instrucción para el proyecto y la ejecución de obras de hormigón en masa y armado, EH 73. Publicada por el M.O.P., Madrid, 1973.

Control de calidad de las obras de hormigón. Capítulo 9 del libro "Hormigón Armado"; por MONTOYAMESEGUER-MORAN. Editado por Gustavo Gili, Madrid, 1973.

Recomendaciones de actuación para mejorar la calidad de la construcción en España. Libro verde prepa. rado por el Comité de Construcción de la Asociación Española para el Control de la Calidad, Madrid, 1973. 


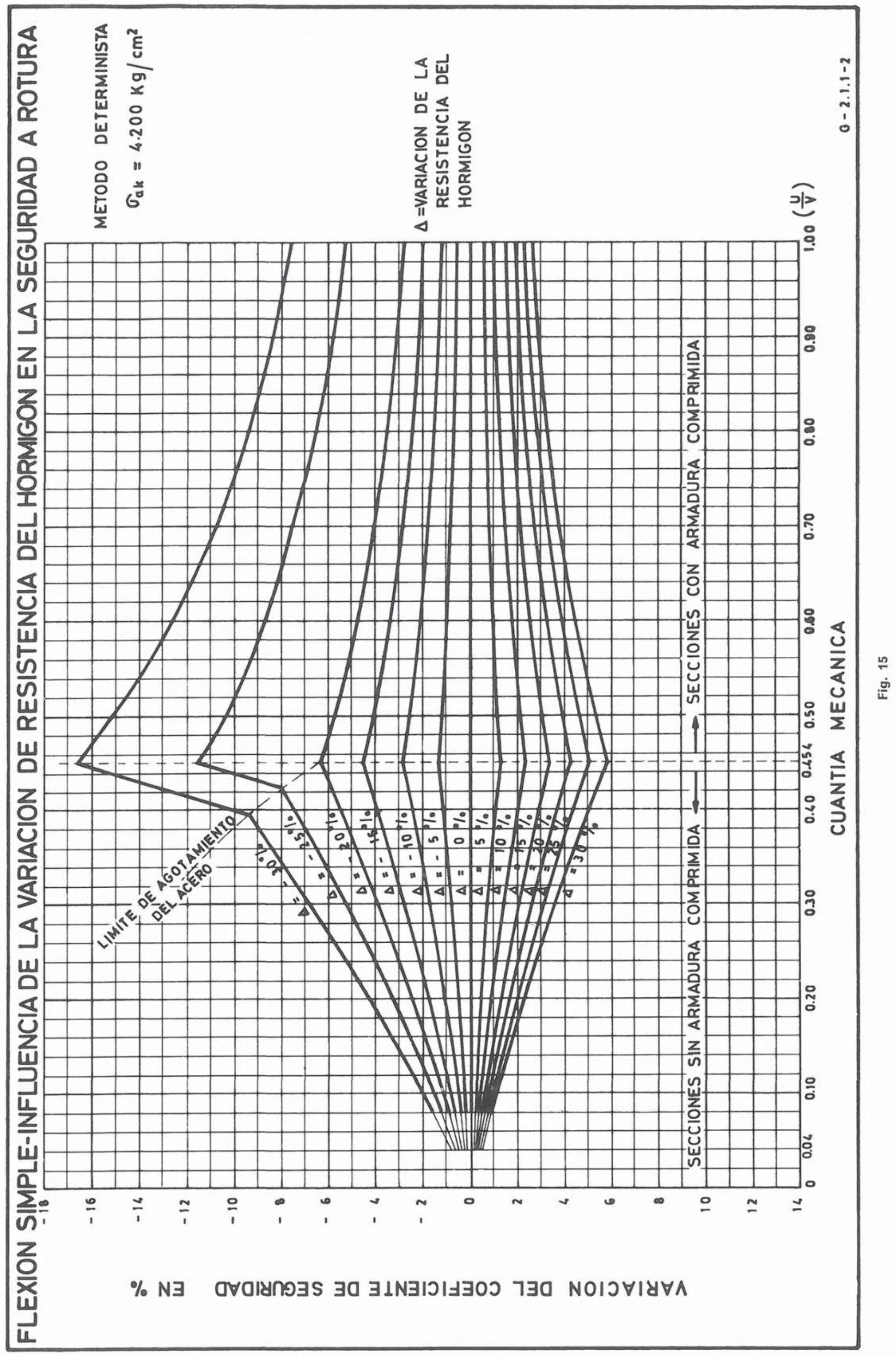

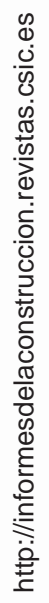

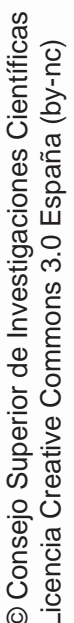



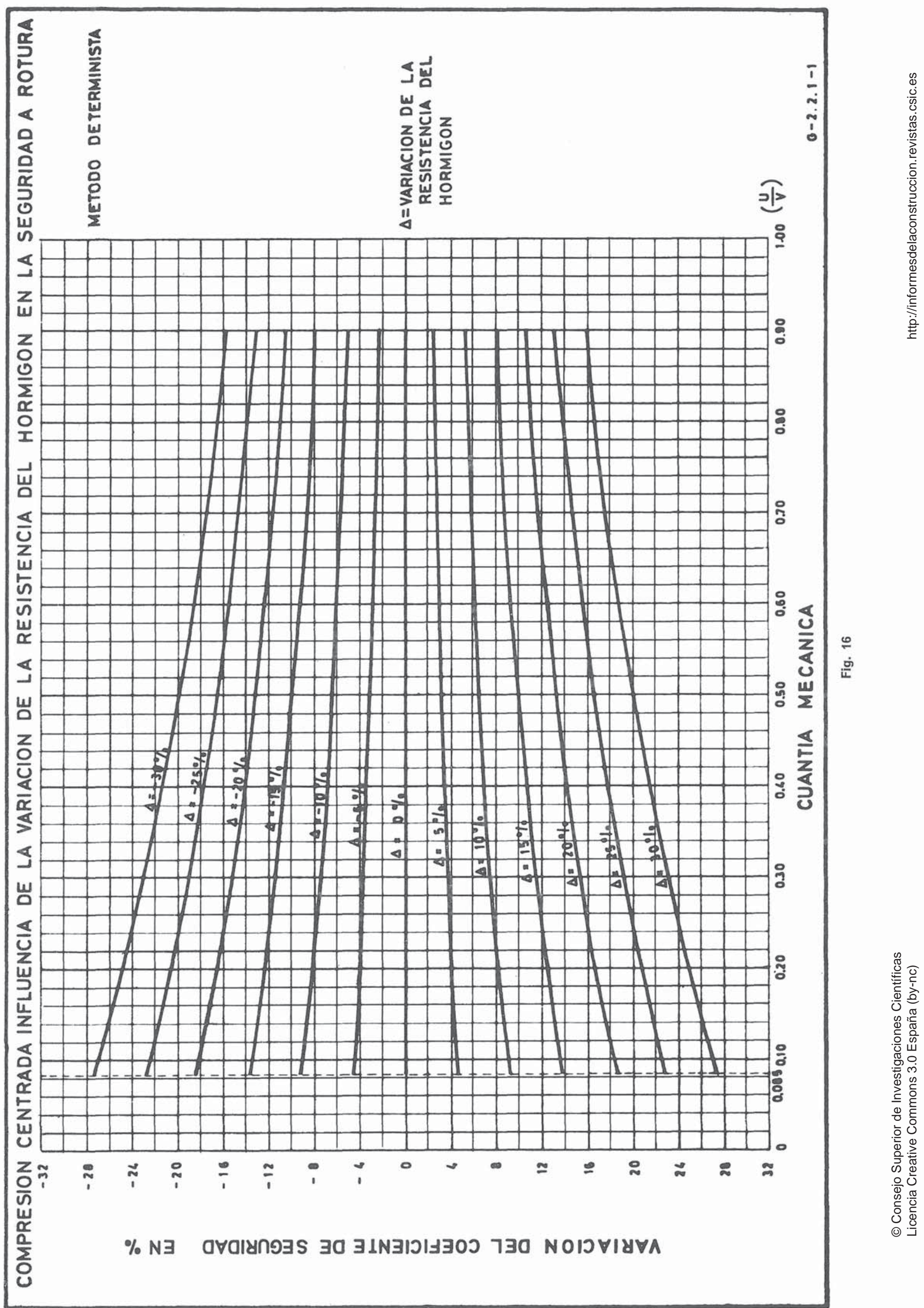


\title{
TOUR D'HORIZON ACTUEL DU CONTROLE DE QUALITE DU BETON
}

Alvaro García Meseguer, Professeur de recherche

L'état actuel du problème est présenté avec certains détails, tenant compte des travaux en cours du Comité Editorial CEB-CIB-FIP-RILEM sur Contrôle Statistique de Qualité du Béton. D'une manière comparative se présente aussi le modus operandi introduit récemment en Espagne par le nouveau Règlement du Béton EH 73.

L'article conclut en commentant l'influence que peut avoir une diminution de la résistance du béton sur la sécurité de la structure, un problème plus complexe de ce qu'il pourrait paraître à première vue.

\section{PRESENT EUROPEAN OUTLOOK ON CONCRETE QUALITY CONTROL}

Alvaro García Meseguer, research professor

The present state of the problem is presented with some detail, taking into account the work in course of the Editorial Committee CEB-CIB-FIP-RILEM on Statistical Quality Control of Concrete. In a comparative way the modus operandi, recently introduced in Spain through the new Code of Concrete EH 73, is also presented.

The paper concludes commenting on the influence which the decrease of concrete strength has on the safety of the structure, a problem which is more complicated thanit might appear at first sight.

\section{GEGENWÄRTIGE EUROPÄISCHE AUSSICHT DER QUALITATSKONTROLLE DES BETONS}

Alvaro García Meseguer, Versuchsprofessor

Dieses Dokument gibt Aufschluss über den aktuellen Zustand des Problems, unter Berücksichtigung der gegenwärtigen Schriften der Arbeitsgruppe CEB-CIB-FIP-RILEM für Statistische Qualitätskontrolle des Betons. Andererseits wird in vergleichender Form der modus operandi dargestellt, der vor kurzem in Spanien mittels der neuen Beton-Vorschrift EH 73 eingeführt wurde.

Der Artikel schliesst mit einer Betrachtung über das Einfluss, der eine Verminderung der Betonfestigkeit auf die Sicherheit des Baues hat, ein Problem, das viel komplizierter ist als es im ersten Moment erscheint.

\section{publicación del i.e.t.c.c. protección química de la construcción}

\author{
HANS KöLZOW \\ Dr. Químico Dipl. \\ Stadtbaurat a.D.
}

La importancia creciente que se concede a la protección química de las obras de fábrica y la carencia de un tratado que reúna toda la literatura dispersa que existe sobre el tema, han Ilevado a la publicación de este libro, que debe leerse con atención en todas y cada una de sus páginas a fin de que nada resulte incomprensible.

En la Technische Akademie de Wuppertal el autor celebró, durante los últimos años, ocho seminarios, de tres días de duración cada uno, sobre protección de obras. Los participantes a estos seminarios han sido arquitectos e ingenieros procedentes de organismos oficiales y de empresas privadas, así como químicos de las industrias dedicadas a la fabricación de sistemas y método químicos protectores. De las conferencias y de los coloquios consiguientes se han obtenido resultados importantes y esperanzadores que merecen ser conocidos por círculos mucho más amplios. Por todo ello se ha creído conveniente la publicación del presente resumen, indicando expresamente que, de ningún modo, se trata de un manual ni de un libro de recetas.

Encuadernado en rústica, de $17 \times 24 \mathrm{~cm}$, compuesto de 74 páginas. Madrid, 1971.

Precios: España: 300 pesetas. Extranjero, $\$ 6$. 